\title{
Performance of boosted object and jet substructure techniques in Run 1 and 2 ATLAS data
}

\author{
Steven Schramm*, on behalf of the ATLAS Collaboration \\ Universite de Geneve ( $\mathrm{CH}$ ) \\ E-mail: steven.schrammecern.ch
}

\begin{abstract}
Hadronic decays of heavy particles with momenta much larger than their mass result in their decay products being reconstructable as a single large-radius jet. The study of the substructure of these jets allows the separation of these boosted decays with respect to more common jets from light-quarks and gluons. Several techniques have been developed by the phenomenology and experimental community to identify jets coming from hadronic decays of boosted top quarks, $\mathrm{W}, \mathrm{Z}$ and Higgs bosons. The performance of several such techniques has been studied in ATLAS using fully-simulated Monte Carlo events, and validated on data using pure samples of top quarks, W bosons from top decays and dijet events. Results of these studies will be presented for Run 1 as well as Run 2 of the LHC.
\end{abstract}

38th International Conference on High Energy Physics

3-10 August 2016

Chicago, USA

${ }^{*}$ Speaker. 


\section{Introduction}

During 2015 and 2016, the LHC delivered proton-proton collisions at a centre of mass energy of $\sqrt{s}=13 \mathrm{TeV}$ and an instantaneous luminosity surpassing the design value of $1 \times 10^{34} \mathrm{~cm}^{-2} \mathrm{~s}^{-1}$. The resulting dataset contains high-energy hard scatter events, including the production of massive Standard Model particles, such as W/Z/H bosons and top quarks. Hadronic decays of these particles produce 2 or 3 quarks respectively, which each hadronize to form a collimated stream of particles. These particle groups deposit energy in the ATLAS [1] calorimeters, and are reconstructed as jets.

While massive particle decays occur back-to-back in the parent particle rest frame, the Lorentz boost to the laboratory frame can present a different picture. If the parent particle has a low $p_{\mathrm{T}}$, then the boost factor is small, and the decay products remain mostly back to back. As the $p_{\mathrm{T}}$ of the parent particle is increased, the boost factor grows correspondingly and thus the decay products become more collimated. Roughly speaking, the angular separation $\Delta R=\sqrt{\Delta \phi^{2}+\Delta \eta^{2}}$ between the decay products of a particle $\rho$ with a mass $m_{\rho}$ and transverse momentum $p_{\mathrm{T}}^{\rho}$ can be related using $\Delta R \approx 2 m_{\rho} / p_{\mathrm{T}}^{\rho}$. For hadronic decays, a small value of $\Delta R$ results in overlapping jets, where the individual decay products cannot be resolved. In this scenario, large-radius (large- $R$ ) jets become a useful way to represent the entire hadronic decay, and thus become a proxy for the parent particle.

The ATLAS Collaboration currently uses large- $R$ jets built using the anti- $k_{t}[2]$ jet clustering algorithm with a distance parameter $R$ of 1.0. The jets are trimmed [3] to remove pileup contributions and then calibrated to account for the non-uniform and non-compensating response of the ATLAS calorimeters to hadronic particles.

\section{Jet mass optimization}

The primary use of large- $R$ jets in ATLAS is for their ability to represent the decay of a single massive particle. One of the most important properties of large- $R$ jets is thus their mass, which should correspond to the mass of the parent particle, thus allowing for the identification of final states resulting from the top quark or W/Z/H boson decays. The ATLAS calorimetry is sufficient to accurately reconstruct the mass of hadronic decays for most jets. However, at very high $p_{\mathrm{T}}$ (very high collimation), the spatial resolution of the calorimeter becomes a limitation. In searches for new physics, these very high $p_{\mathrm{T}}$ jets typically form the most interesting regime of parameter space, thus it is important to find alternative ways of improving the large- $R$ jet mass calculation.

The use of tracks is a logical next step to consider, as the ATLAS tracking detector has much finer spatial resolution than the calorimeters. However, tracks require the presence of a charged particle, and jets contain a sizable fraction of neutral constituents. Calculating a jet mass purely from tracks $\left(m^{\text {track}}\right)$, is thus less effective (worse mass resolution) than calculating a jet mass purely from calorimeter information $\left(m^{\text {calo }}\right)$, even at very high $p_{\mathrm{T}}$, as seen in Figure 1.

Ultimately, the ideal situation is to use the calorimeter energy resolution and the tracker spatial resolution. This can be done using the track-assisted mass $m^{\mathrm{TA}}$, with $m^{\mathrm{TA}}=m^{\text {track }} \cdot p_{\mathrm{T}}^{\text {calo }} / p_{\mathrm{T}}^{\text {track }}$. This scales the track mass, with its superior spatial resolution, by the ratio of calorimeter to track $p_{\mathrm{T}}$, thus fixing the scale of the variable by adding neutral contributions. In Figure 1, this is shown to have a better mass resolution than either the calorimeter or track mass definitions. The trackassisted mass also has a smaller uncertainty [4], making this a powerful new discriminant. 


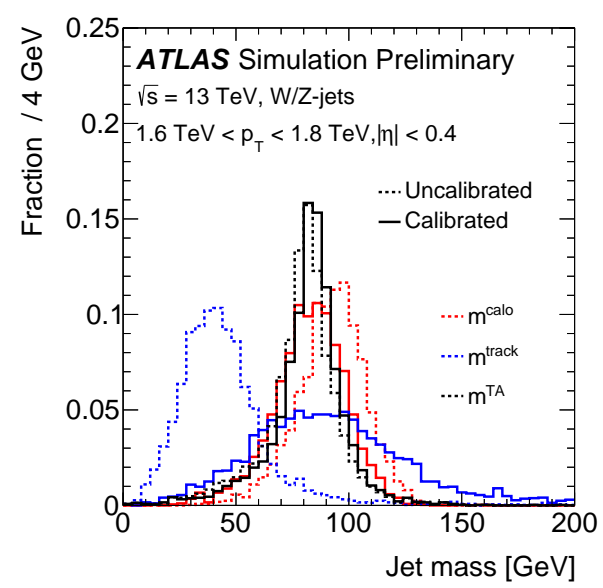

Figure 1: A comparison of hadronically decaying $\mathrm{W}$ and $\mathrm{Z}$ bosons for the normalized calorimeter, track, and track-assisted jet masses $\left(m^{\text {calo }}, m^{\text {track }}\right.$, and $m^{\mathrm{TA}}$ ) for a parent boson $p_{\mathrm{T}}$ between 1.6 and $1.8 \mathrm{TeV}$ [4].

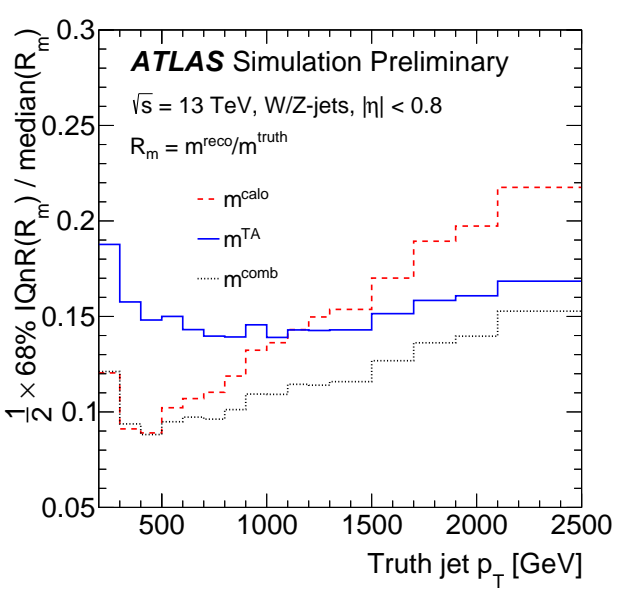

Figure 2: A comparison of hadronically decaying $\mathrm{W}$ and $\mathrm{Z}$ bosons for the calorimeter, track-assisted, and combined jet mass resolutions $\left(m^{\text {calo }}, m^{\mathrm{TA}}\right.$, and $\left.m^{\text {comb }}\right)$ as a function of the parent boson $p_{\mathrm{T}}[4]$.

The track-assisted mass may work very well at high $p_{\mathrm{T}}$, but it is still a quantity which is only accounting for neutral contributions on average. The calorimeter mass can account for localized neutral contributions, and thus it performs better until the calorimeter's spatial resolution is insufficient. This is seen to occur at a $p_{\mathrm{T}}$ of approximately $1.2 \mathrm{TeV}$ for hadronically decaying $\mathrm{W}$ and $\mathrm{Z}$ bosons in Figure 2. Note that this crossing point will depend on the parent particle, as the calorimeter performance depends on the collimation $\Delta R$ which varies with the parent particle mass.

The calorimeter and track-assisted mass each have a regime of parameter space in which they are the best option, and so it is sensible to form a combined mass $m^{\text {comb }}$ from the two variables. This is shown to perform optimally over the full parameter space in Figure 2, and is calculated using weights based on their mass resolutions $\sigma$ :

$$
m^{\text {comb }}=m^{\text {calo }} \cdot \frac{\sigma_{\text {calo }}^{-2}}{\sigma_{\text {calo }}^{-2}+\sigma_{\mathrm{TA}}^{-2}}+m^{\mathrm{TA}} \cdot \frac{\sigma_{\mathrm{TA}}^{-2}}{\sigma_{\text {calo }}^{-2}+\sigma_{\mathrm{TA}}^{-2}} .
$$

\section{Hadronic tagging performance}

In preparation for the $\sqrt{s}=13 \mathrm{TeV}$ dataset, ATLAS has defined new taggers for the hadronic decays of $\mathrm{W} / \mathrm{Z}$ bosons, $\mathrm{H} \rightarrow \mathrm{bb}$, and top quarks. The W/Z boson tagger provides a QCD multijet rejection factor falling roughly linearly from 60 to 30 , as the $p_{\mathrm{T}}$ is varied from $200 \mathrm{GeV}$ to $2 \mathrm{TeV}$, for a flat signal efficiency of 50\% [5]. The $\mathrm{H} \rightarrow$ bb tagger combines b-tagging with substructure information to provide rejection factors of $10^{5}$ (6) for light-quark and gluon jets (bb-jets, such as $\mathrm{g} \rightarrow \mathrm{bb}$ ) for a signal efficiency of roughly $50 \%$ [6]. Tagging of hadronic top decays at a flat $50 \%$ (80\%) signal efficiency provides QCD multijet rejection factors falling roughly linearly from 16 to 8 (5 to 4$)$ as the $p_{\mathrm{T}}$ is varied from $400 \mathrm{GeV}$ to $1.7 \mathrm{TeV}$ [7].

The hadronic $\mathrm{W}$ and top taggers have now been evaluated in situ in 2015 data using a semileptonic $t \bar{t}$ topology, without the application of large- $R$ jet mass selections. The simulated dataset is observed to match the data within the associated uncertainties, as shown in Figure 3, demonstrating 
that the taggers have been properly optimized to identify hadronic decays. Figure 3 also clearly demonstrates that the subsequent application of mass cuts around the $\mathrm{W}$ or top mass peaks will provide further background rejection, leaving a sample relatively pure in the object of interest.
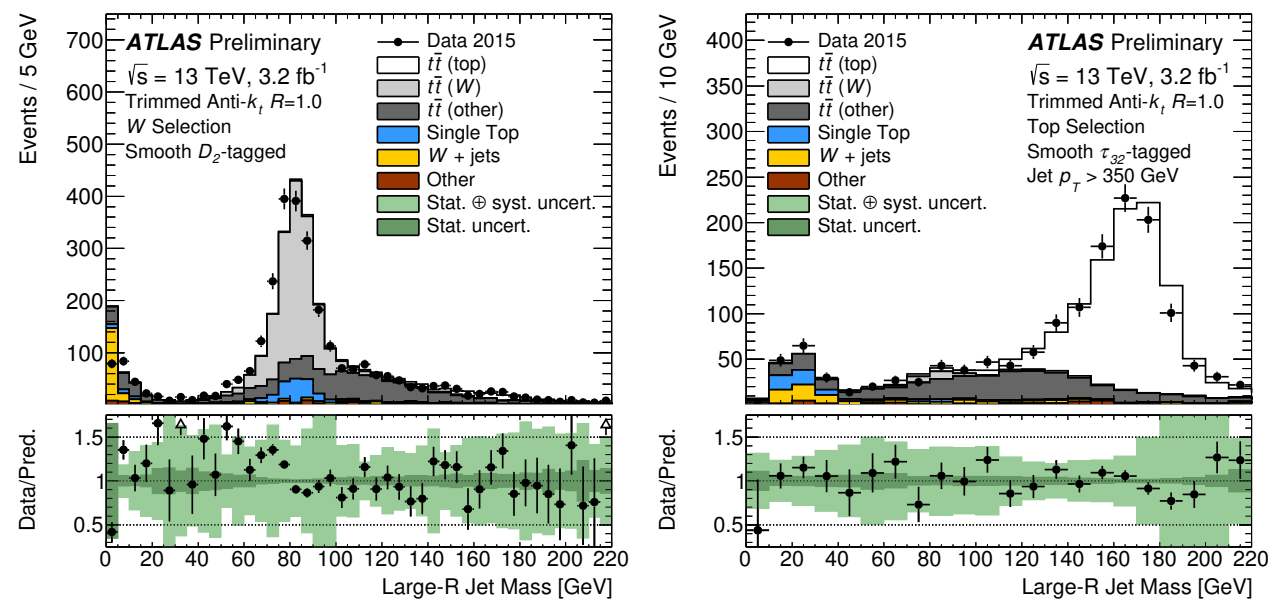

Figure 3: The application of the hadronic $\mathrm{W}$ tagger (left) and top tagger (right) in 2015 data using a semileptonic $t \bar{t}$ topology, before applying a large- $R$ jet mass selection. The simulation and data are observed to agree within the associated uncertainties [8].

\section{Summary}

ATLAS has developed taggers for the hadronic decays of W/Z/H bosons and top quarks, which were optimized for and now evaluated in $\sqrt{s}=13 \mathrm{TeV}$ data. The taggers are performing well, but are less optimal at high $p_{\mathrm{T}}$ due to the limitations of the calorimeter spatial resolution. A new approach to define a mass discriminant using both track and calorimeter information appears very promising, and will be of great use in probing high-energy hadronic decays in the years to come.

\section{References}

[1] ATLAS collaboration, ATLAS Experiment at the CERN Large Hadron Collider, JINST 3 (2008) 003.

[2] M. Cacciari, G. P. Salam and G. Soyez, The Anti-k(t) jet clustering algorithm, JHEP 04 (2008) 063, [0802.1189].

[3] D. Krohn, J. Thaler and L.-T. Wang, Jet Trimming, JHEP 02 (2010) 084, [0 912 . 1342 ].

[4] ATLAS collaboration, Jet mass reconstruction with the ATLAS Detector in early Run 2 data, ATLAS-CONF-2016-035 (2016).

[5] Identification of boosted, hadronically-decaying $W$ and $Z$ bosons in $\sqrt{s}=13$ TeV Monte Carlo Simulations for ATLAS, ATL-PHYS-PUB-2015-033 (2015).

[6] Expected Performance of Boosted Higgs $(\rightarrow b \bar{b})$ Boson Identification with the ATLAS Detector at $\sqrt{s}=13 \mathrm{TeV}$, ATL-PHYS-PUB-2015-035 (2015).

[7] Boosted hadronic top identification at ATLAS for early 13 TeV data, ATL-PHYS-PUB-2015-053 (2015).

[8] ATLAS Collaboration, "Public Plots for Top and Boson Tagger Performance." http://atlas.web.cern.ch/Atlas/GROUPS/PHYSICS/PLOTS/JETM-2016-005/. 